\title{
Detailed Simulations of Shock-Bifurcation and Ignition of an Argon-diluted Hydrogen/Oxygen Mixture in a Shock Tube
}

\author{
Matthias Ihme, Yong Sun ${ }^{\dagger}$ \\ Department of Aerospace Engineering, University of Michigan, Ann Arbor, MI 48109 \\ and Ralf Deiterding \\ Oak Ridge National Laboratory, Oak Ridge, TN 37831
}

\begin{abstract}
Detailed simulations of the bifurcation and ignition of an Argon-diluted Hydrogen/Oxygen mixture in the two-stage weak ignition regime are performed. An adaptive meshrefinement (AMR) technique is employed to resolve all relevant physical scales that are associated with the viscous boundary-layer, the reaction front, and the shock-wave. A high-order hybrid WENO/central-differencing method is used as spatial discretization scheme, and a detailed chemical mechanism is employed to describe the combustion of the $\mathrm{H}_{2} / \mathrm{O}_{2}$ mixture. The operating conditions considered in this study are $p_{5}=5$ bar and $T_{5}=1100 \mathrm{~K}$, and fall in the third explosion limit. The computations show that the mixing of the thermally stratified fluid, carrying different momentum and enthalpy, introduces inhomogeneities in the core-region behind the reflected shock. These inhomogeneities act as localized ignition kernels. During the induction period, these kernels slowly expand and eventually transition to a detonation wave that rapidly consumes the unburned mixture. In competition with this detonation wave are the presence of secondary ignition kernels that appear in the unreacted core-region between reflected shock and detonation wave.
\end{abstract}

\section{Introduction}

$\mathrm{T}$ HE accurate description of combustion chemistry and chemical-kinetic models is critical for characterizing effects of new fuel compositions on existing propulsion systems and for developing future combustion technologies. Among other facilities, shock tubes remain hereby invaluable in providing detailed information about ignition delay times, extinction limits, and species time-histories for the development and validation of reaction mechanisms. In its simplest form, a shock tube consists of a constant-diameter tube, which is divided into a driver section and a driven section by a diaphragm. While the driver section is pressurized with an inert gas, the driven section contains the diluted test gas mixture that is under experimental investigation. Following the rapture of the diaphragm, a normal shock develops which propagates into the driven section and is reflected at the end-wall. Under ideal conditions, the test gas mixture in the region behind the reflected shock is stationary and uniform. While this condition is ideal for chemical-kinetics investigations, practical shock tube systems are affects by non-idealities. Over recent years, several sources responsible for non-ideal shock tube behaviors have been identified, including the non-ideal rapture of the diaphragm, ${ }^{1-5}$ boundary layer growth, ${ }^{6-8}$ reflected shock/boundary layer interactions, ${ }^{9-15}$ and inhomogeneous ignition and weak-tostrong ignition transition. ${ }^{16-24}$ The direct consequence of these non-idealities are increasing uncertainties in the measurements that are reflected by errors in derived rate coefficients and ignition delay times. ${ }^{25}$

Of particular interest to the shock-tube community is the state behind the reflected shock. Inviscid and one-dimensional theory shows that the mixture is stagnant after the shock reflects, providing ideal homogeneous conditions for chemical-kinetics investigations. However, under realistic conditions, the reflected

*Assistant Professor, AIAA member; correspondence: mihme@umich.edu

$\dagger$ Graduate Student

${ }^{\ddagger}$ Research Scientist 
shock interacts with the boundary layer as it moves away from the wall. Through this interaction the shock bifurcates, which leads to the development of strong velocity currents that penetrate through the bifurcated shock foot into the test gas region, introducing velocity fluctuations and non-uniformities in the mixture composition. ${ }^{9}$ The direct consequence of the boundary layer growth on the observed thermodynamic quantities behind the reflected shock is a constant drift in temperature and pressure. In particular, the increase in temperature due to boundary layer growth has dramatic effects on the measurements of activation energies and ignition delay times, and can result in systematic errors in excess of $20-40 \%{ }^{6,26}$ Further, residual gas motion behind the reflected shock can also contribute to local pressure inhomogeneities. ${ }^{27}$ Although it has recently been recognized that these effects require consideration, ${ }^{7,28,29}$ a satisfactory theoretical characterization has so far not been established. This is primarily due to the fact that the boundary layer interaction and shock bifurcation occur in the transitional and turbulent flow regime and are largely dependent on geometry and conditions behind and in front of the shock. ${ }^{30}$ For practical applications, boundary layer effects can be partially mitigated by employing large shock tube diameters and reducing the test time. ${ }^{26}$ However, if the shock tube operating range is extended to higher conversion rates, larger pressures, and longer ignition delay times, boundary layer and bifurcation will increase in importance, and result in the loss of experimental accuracy. ${ }^{29}$ As such, the direct and indirect effect of the boundary layer on the condition and mixture behind the reflected shock represents a limiting factor towards extending the shock tube performance to longer operating conditions.

The objective of this work is to address the issue of boundary-layer induced shock-tube non-idealities, and to characterize effects of the shock-bifurcation on the flow-field and ignition dynamics in the region behind the reflected shock. To this end, detailed numerical simulations at relevant operating conditions will be conducted in order to quantify direct contributions of the boundary layer development on the incident shock wave and resulting deviations from ideal shock tube conditions.

The remainder of this paper is outlined as follows. A physical description of the shock bifurcation is presented in the next section. The mathematical model, AMR-method, and details of the numerical methods are discussed in Sec. III. The computational configuration, selection of operating conditions, and numerical setup are presented in Sec. IV. Simulation results for non-reacting and reacting configurations are separately discussed in Secs. V.A and V.B, and the paper finishes with conclusions in Sec. VI.

\section{Physics of Shock Bifurcation}

Shock bifurcation is one of the major mechanisms responsible for the non-ideal behavior in shock tube facilities. An instantaneous toluene PLIF-image together with a schematic of a shock-bifurcation are shown in Fig. 1. The structure of a bifurcated shock consists of a bifurcation foot, a tail-shock, and a triple point at which the bifurcated foot and tail shocks merge and from which the slip line originates.

A shock-bifurcation is formed when a reflected shock interacts with the low-momentum boundary layer that is developed behind the incident shock as it propagates toward the end-wall. Under the condition that the stagnation pressure in the boundary-layer is lower than that in the region behind the reflected shock, the boundary-layer does not remain attached and separates from the wall as it is ingested by the upstreampropagating shock-wave. The flow eventually stagnates and reverses direction, resulting in the formation of a viscous layer that is carried along with the upstream-propagating shock foot. Due to the difference in pressure and entropy across the normal reflected shock and the bifurcation region a high-momentum jet develops as the boundary-layer fluid is deflected and passes through the oblique shock. As the fluid is ejected at the tail shock, a shear-layer develops between the shock-compressed and colder boundary-layer fluids. The resulting slip line separates the stagnant flow behind the normal shock and the flow that is accumulated in the bifurcation zone. This slip line is hydro-dynamically unstable and promotes mixing between the different fluids. This instability is further amplified by the pressure difference across the shear-layer as a result of the recompression by the tail shock. The mixing in the shear-layer is complex and involves the interaction among three thermally stratified fluid layers: ${ }^{31}$ The lower layer at the wall consists of boundary-layer fluid in the recirculation zone. Above this is the fluid which is entrained through the bifurcation foot and tail shocks, and the third layer consists of the hot shock-compressed fluid in the core. With increasing distance away from the bifurcation the fluid mixture equilibrates and the slip line breaks up.

As the boundary-layer is ingested by the reflected shock, the adverse pressure gradient across the bifurcation causes it to separate and eventually stagnate. The entrainment of fluid through the bifurcation foot results in a continuous growth of the triple-shock structure. Although the topology of this structure re- 

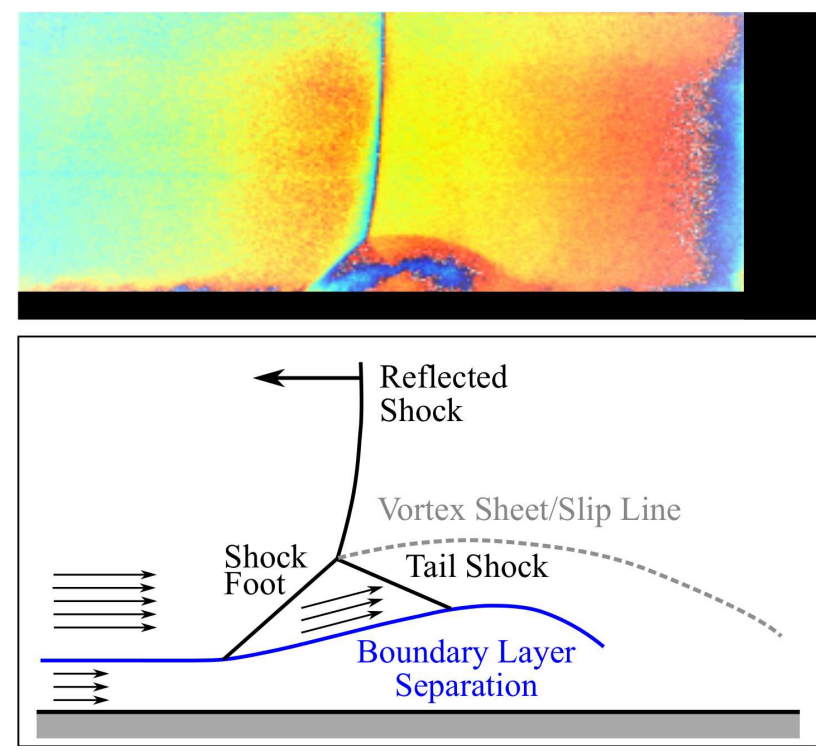

Figure 1. Shock bifurcation; top: toluene PLIF measurements (conditions: $(T, p)_{1}=\left(296 \mathrm{~K}, 32\right.$ torr), $(T, p)_{2}=$ $(498 \mathrm{~K}, 0.25 \mathrm{~atm}),(T, p)_{5}=(696 \mathrm{~K}, 1.05 \mathrm{~atm}), X=8 \%$ Toluene $/ \mathrm{N}_{2}$; image courtesy: R. Hanson); bottom: schematic of shock-bifurcation structure.

mains unaffected, the bifurcation structure increases in size at an early stage until an equilibrium is reached. Furthermore, the continuous entrainment of the boundary layer increases the size of the interaction zone, thereby further enhancing the mixing in the region behind the reflected shock.

The shock bifurcation structure was first observed experimentally by Mark ${ }^{9}$ and Strehlow \& Cohen. ${ }^{10}$ Mark $^{9}$ developed a theoretical model for describing the onset and structure of the shock-bifurcation. Over recent years different experimental techniques (Schlieren, Laser Doppler velocimetry, pressure measurements, interferometry, toluene-PLIF, Hydroxyl tagging velocimetry) have been applied to further characterize the flow-field behind reflected and incident shocks ${ }^{32,33}$ and the shock-bifurcation structure. ${ }^{10,11,15,34-36}$ Figure 1 (top) shows an instantaneous toluene-PLIF image of a bifurcation-structure. Clearly evident from this image is the curved structure of the reflected shock, the bifurcated foot and tail shocks that merge with the main reflected shock at the triple point from which the slip line originates. Also evident is the separation of the colder boundary layer fluid as it passes underneath the oblique shock.

\section{Mathematical Model}

\section{A. Governing Equations}

The governing equations that are employed to describe the spatio-temporal evolution of the flow, ignition, and combustion inside the shock tube are the reactive Navier-Stokes equations, which are here written in index form as:

$$
\frac{\partial \mathbf{U}}{\partial t}+\frac{\partial}{\partial x_{j}}\left(\mathbf{F}_{j}^{c}-\mathbf{F}_{j}^{v}\right)=\mathbf{S}
$$

where $\mathbf{U}$ is the state-vector, $\mathbf{F}_{j}^{c}$ and $\mathbf{F}_{j}^{v}$ are the convective and viscous fluxes, and $\mathbf{S}$ is the source term vector. These quantities have the following definition:

$$
\begin{aligned}
\mathbf{U} & =\left(\rho u_{i}, \rho e_{t}, \rho Y_{n}\right)^{T}, \\
\mathbf{F}_{j}^{c} & =\left(\rho u_{j} u_{i}+p \delta_{i j}, u_{j}\left(\rho e_{t}+p\right), \rho Y_{n} u_{j}\right)^{T}, \\
\mathbf{F}_{j}^{v} & =\left(\tau_{i j}, u_{i} \tau_{i j}+q_{j}, \rho Y_{n} V_{j n}\right)^{T}, \\
\mathbf{S} & =\left(0_{i}, 0, \dot{\omega}_{n}\right)^{T},
\end{aligned}
$$


for $\{i, j, k\}=1, \ldots, N_{D}$ and $\{n, m\}=1, \ldots, N_{S}$, where $N_{D}$ denotes the spatial dimension and $N_{S}$ is the number of chemical species. The total internal energy is:

$$
e_{t}=\sum_{n=1}^{N_{S}} Y_{n} h_{n}-\frac{p}{\rho}+\frac{1}{2} u_{i}^{2} .
$$

The viscous stress tensor and species diffusion velocity are written as:

$$
\begin{aligned}
\tau_{i j} & =\mu\left(\frac{\partial u_{i}}{\partial x_{j}}+\frac{\partial u_{j}}{\partial x_{i}}\right)-\frac{2}{3} \mu \frac{\partial u_{k}}{\partial x_{k}} \delta_{i j}, \\
V_{j n} & =\frac{1}{X_{n} \bar{W}} \sum_{m \neq n}^{N_{S}} W_{m} D_{n, m} d_{j m}-\frac{D_{n}^{T}}{\rho Y_{n}} \frac{\partial \ln T}{\partial x_{j}},
\end{aligned}
$$

where $D_{n, m}$ and $D_{n}^{T}$ are the multicomponent diffusion and thermal diffusion coefficients, respectively, and

$$
d_{j m}=\frac{\partial X_{m}}{\partial x_{j}}+\left(X_{m}-Y_{m}\right) \frac{\partial \log p}{\partial x_{j}} .
$$

The heat flux vector, appearing in Eq. (2c), is evaluated as:

$$
q_{j}=\sum_{n=1}^{N_{S}} \rho Y_{n} V_{j n} h_{n}-\lambda \frac{\partial T}{\partial x_{j}}-\sum_{n=1}^{N_{S}} \frac{\mathcal{R} T}{W_{n} X_{n}} D_{n}^{T} d_{j n},
$$

where $\lambda$ is the thermal conductivity.

To close the system of equations, Eq. (2) is supplemented by the ideal gas law, which is written as:

$$
p=\rho \mathcal{R} T \sum_{n=1}^{N_{S}} \frac{Y_{n}}{W_{n}}
$$

where $\mathcal{R}$ is the universal gas constant and $W_{n}$ is the molecular weight of species $n$. The mole fraction $X_{n}$ is related to the mass fraction via the expression:

$$
X_{n}=\frac{Y_{n} \bar{W}}{W_{n}} .
$$

\section{B. Adaptive Mesh Refinement}

In order to supply the required temporal and spatial resolution efficiently, we employ a block-structured adaptive mesh refinement (AMR) method after Berger \& Colella. ${ }^{37}$ The AMR method is implemented in the object-oriented framework AMROC ${ }^{38}$ (Adaptive Mesh Refinement in Object-oriented $\mathrm{C}++$ ). The code is fully parallelized for distributed memory machines using MPI-libraries.

\section{1. $\quad$ Structured AMR Method}

Instead of replacing single cells by finer ones, as it is done in cell-oriented refinement techniques, structured AMR methods utilize a patch-oriented approach. Cells that are flagged by various error indicators (shaded in Fig. 2(a)) are clustered with a special algorithm ${ }^{39}$ into non-overlapping rectangular grids. In the AMR method, a sequence of $l$ grid-levels is constructed by recursively refining the coarsest grid in regions of interest (see Fig. 2(a)). The spatial and temporal discretization widths on level $l$ are $r_{l}$-times finer than on level $l-1$, i.e. $\Delta t_{l}:=\Delta t_{l-1} / r_{l}$ and $\Delta x_{n, l}:=\Delta x_{n, l-1} / r_{l}$ with $r_{l} \geq 2$ for $l>0$ and $r_{0}=1$, ensuring that a time-explicit finite volume scheme (in principle) remains stable on all levels of the hierarchy.

The numerical scheme is applied on level $l$ by calling a single-grid routine in a loop over all subgrids. The subgrids become computationally decoupled by employing additional ghost cells around each computational grid. Three types of ghost cells have to be considered in the sequential case, see Fig. 2(b). Cells outside of the root domain are used to implement physical boundary conditions. Ghost cells overlaid by a grid on level $l$ have a unique interior cell analogue and are set by copying the data value from the grid, where the interior 


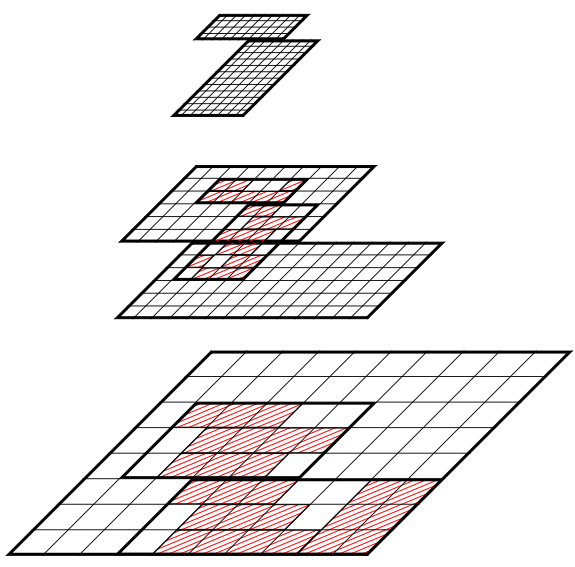

(a) AMR grid-hierarchy.

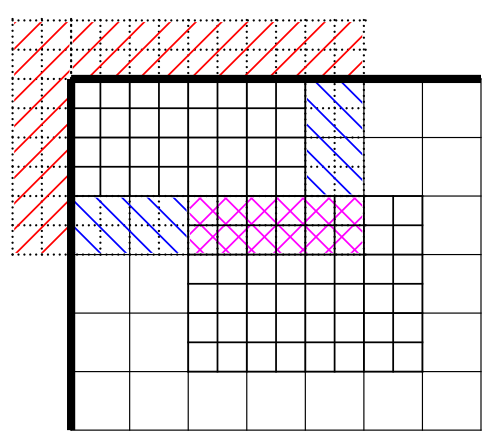

Interpolation Synchronization Physical boundary

(b) Ghost cell definition.

Figure 2. Adaptive mesh-refinement: (a) successively embedded rectangular subgrid topology and (b) ghost cell definition in AMR subgrid.

cell is contained (synchronization). On the root level no further boundary conditions need to be considered, but for $l>0$ also internal boundaries can occur. They are set by a conservative time-space interpolation from two previously calculated time steps of level $l-1$.

Beside a general data tree that stores the topology of the hierarchy, the AMR method requires at most two regular arrays assigned to each subgrid. They contain the discrete state-vector for the actual and updated time step. The regularity of the data allows high performance on vector and super-scalar processors and cache optimizations. Small data arrays are effectively avoided by leaving coarse-level data-structures untouched when higher level grids are created. Values of cells covered by finer subgrids are overwritten by averaged fine grid values subsequently. This operation leads to a modification of the numerical stencil on the coarse mesh and requires a special flux correction in cells abutting a fine grid. The correction replaces the coarse grid flux along the fine grid boundary by a sum of fine fluxes and ensures the discrete conservation property of the hierarchical method at least for purely Cartesian problems without embedded boundaries. ${ }^{37,40}$

\section{Parallelization}

The AMROC framework utilizes a rigorous domain decomposition approach, in which the subdomain partitioning is controlled at the root-level. The key idea is that all higher level domains are required to follow this "floor-plan." A careful analysis of the AMR algorithm uncovers that the only parallel operations under this paradigm are ghost cell synchronization, redistribution of the AMR hierarchy, and the application of the previously mentioned flux correction terms. On the other side, computational complex operations such as interpolation, averaging, and the calculation of flux corrections remain strictly local. ${ }^{40,41}$ Currently, a generalization of Hilbert's space-filling curves ${ }^{42}$ is employed to obtain load-balanced root level distributions at runtime. The entire AMR hierarchy is considered by projecting the accumulated work from higher levels onto the root level cells. Although rigorous domain decomposition does not lead to a perfect balance of workload on single levels, good scalability up to $\mathcal{O}\left(10^{3}\right)$ CPU cores is typically achieved. AMROC's hierarchical data structures are derived from the DAGH (Distributive Adaptive Grid Hierarchies) package ${ }^{43}$ and are implemented completely in $\mathrm{C}++$.

\section{Numerical Method and Hybrid WENO/CD Scheme}

The shock-capturing component of the present hybrid schemed uses a sixth-order accurate, symmetric enhancement of the classical WENO scheme as developed by Shu. ${ }^{44,45}$ In the limit of a smooth flow, the method obtains a 6th-order centered stencil. Hill et al. ${ }^{46}$ and Pantano et al. ${ }^{47}$ developed a robust hybrid WENO/tuned centered difference (TCD) method, which combines the TCD stencil with the WENO-SYM scheme. The centered difference stencil was bandwidth-optimized, specifically for weakly compressible decaying turbulence. ${ }^{47}$ The optimal WENO weights are chosen to match those of the TCD scheme thereby 
minimizing oscillations at the matching boundaries. The location of the scheme-switching boundary is defined by a problem-dependent switch. By using the relatively inexpensive TCD stencil predominantly in regions where the solution is smooth and WENO-SYM at and around discontinuities, the overall resulting WENO/TCD scheme performs better and additionally has the spectral resolution desired for turbulence simulations. For DNS, however, where all scales are resolved, a symmetric order-optimized stencil is ideal. Therefore, for our application a WENO/CD rather than WENO/TCD method is used. For schemes based on centered stencils, no numerical viscosity is present, yet care is needed to avoid nonlinear instabilities that may develop. ${ }^{47}$ Such instabilities can be alleviated by using a skew-symmetric formulation that conserves the kinetic energy ${ }^{48}$ and prevents the convective terms of the momentum and energy equations from artificially producing or dissipating global kinetic energy. Without this, it has been found that in unstable flow simulations, the entropy of the system decreases with time, violating the second law of thermodynamics.

The WENO/CD scheme requires an explicit switch, from regions of smooth flow (using CD) to regions with strong gradients that require WENO-limiting. The shock-based detection method ${ }^{49}$ that is employed in this contribution uses an approximate Riemann solver to detect the existence and orientation of strong shock waves, while ignoring weak ones. The approximate solution to the Riemann problem is computed using Roe-averaged quantities from the given left $(L)$ and right $(R)$ state. Liu's entropy condition allows for characterizing the type of wave encountered at the characteristic associated with the eigenvalues $u \pm a$ (shock or rarefaction wave). A shock is produced if and only if the central state satisfies the condition:

$$
\left|u_{R} \pm a_{R}\right|<\left|u_{*} \pm a_{*}\right|<\left|u_{L} \pm a_{L}\right| \text {. }
$$

Here, $a_{L, R}$ is computed by evaluating the speed of sound, $a=\sqrt{\gamma p / \rho}$, at the left or right cell faces, and the central state $\left(u_{*}, a_{*}\right)$ corresponds to the Roe averages,

$$
u_{*}=\frac{\sqrt{\rho_{L} u_{L}}+\sqrt{\rho_{R} u_{R}}}{\sqrt{\rho_{L}}+\sqrt{\rho_{R}}}, \quad a_{*}=\sqrt{\frac{1}{2}\left(\gamma_{*}-1\right)\left(2 h_{*}-u_{*}^{2}\right)},
$$

where

$$
\begin{aligned}
h_{*} & =\frac{\sqrt{\rho_{L} h_{L}}+\sqrt{\rho_{R} h_{R}}}{\sqrt{\rho_{L}}+\sqrt{\rho_{R}}}, \\
c_{p, *} & =\frac{\sqrt{\rho_{L} c_{p, L}}+\sqrt{\rho_{R} c_{p, R}}}{\sqrt{\rho_{L}}+\sqrt{\rho_{R}}}, \\
\gamma_{*} & =\frac{c_{p, *}}{c_{p, *}-R_{*}}, \\
R_{*} & =\frac{\sqrt{\rho_{L} R_{L}}+\sqrt{\rho_{R} R_{R}}}{\sqrt{\rho_{L}}+\sqrt{\rho_{R}}},
\end{aligned}
$$

and $h_{*}, \gamma_{*}, c_{p, *}$, and $R_{*}$ are the Roe-averaged specific enthalpy, ratio of specific heats, specific heat at constant pressure, and gas constant, respectively. When testing the validity of the inequalities (9), a threshold value $\alpha_{\text {Liu }} / a$ is considered to eliminate weak acoustic waves that could be easily handled by the CD scheme. For the present application $\alpha_{\text {Liu }}$ takes a values of 0.01. For better efficiency and flexibility, this criterion is combined with a geometrical test that is based on a mapping of the normalized pressure gradient, $\theta_{j}$, that reads $^{49}$

$$
\phi\left(\theta_{j}\right)=\frac{2 \theta_{j}}{\left(1+\theta_{j}\right)^{2}} \quad \text { with } \quad \theta_{j}=\frac{\left|p_{j+1}-p_{j}\right|}{\left|p_{j+1}+p_{j}\right|} .
$$

If Eq. (9) is satisfied for a cell that differs by surrounding cells with values different by at least $\alpha_{L i u} / a$ and also $\phi\left(\theta_{j}\right)>\alpha_{\text {Map }}$ holds true, then WENO is set to be used at the cell wall. The threshold $\alpha_{\text {Map }}$ is set to 0.005. This algorithm is applied independently in each spatial direction and we additionally employ it in rotated frames to detect shocks that are not grid aligned.

In here, we employ WENO and CD numerical fluxes that are both six-order accurate in space. The temporal update of the hybrid WENO/CD scheme is performed using a strong stability preserving explicit three-stage Runge-Kutta scheme yielding an overall numerical method of formally 3rd order accuracy. 


\begin{tabular}{|c|c|c|c|}
\hline Level $l$ & $r_{l}$ & $\Delta x[\mathrm{~mm}]$ & $\Delta y[\mathrm{~mm}]$ \\
\hline \hline 0 & 1 & 0.5 & 0.2 \\
\hline 1 & 2 & 0.25 & 0.1 \\
\hline 2 & 2 & 0.125 & 0.05 \\
\hline 3 & 2 & 0.0625 & 0.025 \\
\hline 4 & 4 & 0.015625 & 0.00625 \\
\hline
\end{tabular}

Table 1. AMR setup and grid-resolution.

\section{Computational Configuration}

The objective of this investigation is to study the ignition of diluted hydrogen/oxygen mixtures at operating conditions that are relevant to gas turbine engines. These conditions correspond to pressures above 5 bar and temperatures between 650 and $1100 \mathrm{~K}$, corresponding to the third explosion limit. However, it was experimentally observed ${ }^{10,18,20,21,50-52}$ (see also review-article by Chaos \& Dryer ${ }^{53}$ ) that ignition behind a reflected shock exhibits different characteristics that are referred to as "weak" or "strong" ignition regime. At sufficiently low temperature, weak ignition is initiated by localized ignition kernels in the wall-near region. These kernels eventually transition and merge to a fully developed flame front. The chemical induction time in this weak ignition regime is particularly sensitive to stratification and conditions of the mixture, and often observed as stochastic event. On the other side, strong ignition is observed at higher shock-reflection temperatures. In this scenario, a localized ignition spot transitions instantaneously to a detonation wave, which is accompanied by a rapid increase in pressure and temperature.

To computationally investigate the ignition-process, operating conditions are selected that are consistent with conditions that have been previously considered in experimental investigations by Blumenthal et al. ${ }^{18,51}$ and Pang et al. ${ }^{54}$ To this end, an Argon-diluted Hydrogen/Oxygen mixture with the molar ratio: $X_{\mathrm{H}_{2}} / X_{\mathrm{O}_{2}} / X_{\mathrm{Ar}}=15 / 17.85 / 67.15$ is considered. The reaction chemistry is described by the updated high-pressure $\mathrm{H}_{2} / \mathrm{O}_{2}$ kinetic mechanism of Burke et al. ${ }^{55}$ consisting of 13 chemical species and 27 chemical reactions. Initial conditions of the test-gas mixture in the driven and driver sections are chosen to achieve a nominal reflected shock condition of $p_{5}=5$ bar and $T_{5}=1100 \mathrm{~K}$. All simulations are conducted using adiabatic boundary conditions, so that $\partial_{n} T=0$.

Instead of simulating the entire shock tube, in this study we only consider the shock-tube end section. The flow is initialized by a moving shock using the normal shock-relation. The corresponding conditions in the driver and driven sections are:

$$
\begin{array}{cl}
\text { Driver Section: } & T_{2}=651 \mathrm{~K}, p_{2}=1.391 \mathrm{bar}, v_{2}=485 \mathrm{~m} / \mathrm{s}, \\
\text { Driven Section: } & T_{1}=304 \mathrm{~K}, p_{1}=0.234 \mathrm{bar}, v_{1}=0 \mathrm{~m} / \mathrm{s}, .
\end{array}
$$

The computational domain consist of a symmetric two-dimensional planar shock-tube, having a length of $20 \mathrm{~cm}$ and a half-height of $1 \mathrm{~cm}$, and five levels of refinement are considered. The computational grid at the coarsest level consists of $400 \times 50$ grid points in stream-wise and wall-normal direction, and the refinement levels and corresponding grid-resolutions are summarized in Tab. 1. An example of an instantaneous flowfield and the corresponding mesh-arrangement is illustrated in Fig. 3, showing axial velocity (top) and AMR-levels (bottom). It can be seen that the finest ARM-level is confined to a localized region surrounding the reflected shock and the separation zone beneath the bifurcated shock region.

\section{Results}

\section{A. Non-reacting Shock Bifurcation}

Computational results for the non-reacting simulation for four different time-instances are presented in Fig. 4, showing the axial velocity fields on the left and the corresponding temperature on the right. Simulation results are shown after the shock is reflected at the end wall. Results in Fig. 4(a) show the viscous boundary layer that is formed behind the incident shock wave. The viscous dissipation increases the temperature inside the boundary-layer, which is approximately $150 \mathrm{~K}$ higher than in the core-region of the shock-tube. It will 


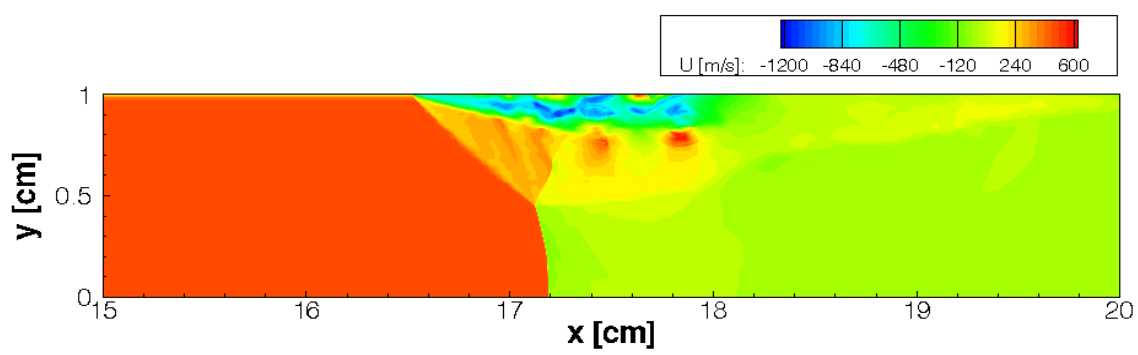

(a) Axial velocity.

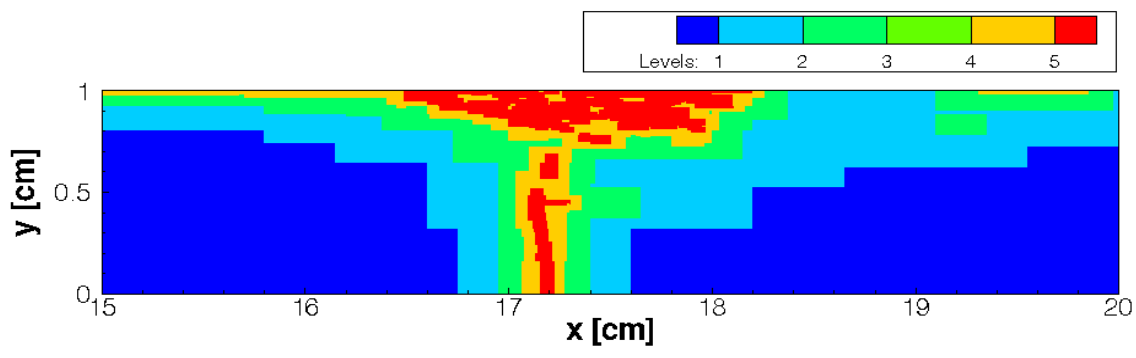

(b) AMR refinement levels.

Figure 3. Illustration of mesh-refinement showing (a) axial velocity and (b) AMR-levels for reacting flow configuration at time $t=67.5 \mu \mathrm{s}$.

be shown in the next section that the viscous heating in conjunction with the oblique shock-compression introduces localized ignition kernels in the bifurcation region, acting as primary ignition sources.

From Fig. 4(a) it can be seen that the bifurcation develops instantaneously after the shock is reflected at the end-wall. Clearly visible are the bifurcation foot and tail shocks, and the boundary layer separation as the fluid passes underneath the bifurcation foot into the high-pressure region behind the reflected shock. The slip line, demarcating the boundary-layer region and stagnant core, is also evident. It can be seen that, initially, the slip-line terminates at the upper corner of the shock-tube. However, with increasing time (see Figs. 4(c) and $4(\mathrm{~d})$ ) the slip-line breaks up due to the formation of a shear-layer instability between the stagnant fluid in the core and the boundary layer. This instability promotes the entrainment of colder boundary layer fluid into the region behind the reflected shock, resulting in the development of a thermally inhomogeneous region that continuously increases in size. This is further illustrated in Fig. 5, showing profiles of velocity, temperature, and pressure along three radial locations through the shock-tube: $y=0.99 \mathrm{~cm}$ (boundary layer), $y=0.5 \mathrm{~cm}$ (middle of the shock tube), and $y=0 \mathrm{~cm}$ (along the shock-tube center). Most notable are the pronounced fluctuations in the boundary layer region (see top row in Fig. 5). However, with increasing distance away from the wall, these inhomogeneities decrease, and the core-region on the inner-side of the slip-line remains initially unaffected by these mixing processes. This is confirmed by the axial profiles along the centerline in the bottom row of Fig. 5. As time progresses and the reflected shock propagates further upstream, the bifurcation region continuously increases in size, which is coupled with an enhanced entrainment of fluid upstream of the shock-compressed region. With this, the boundary layer separation under the bifurcation increases in length and the separation moves further away from the wall. The increasing bifurcation region, however, reduces the size of the homogeneous and unperturbed core-region. Furthermore, it can also be seen that the region behind the normal shock becomes increasingly affected by the expansion of the bifurcation. This is illustrated in the bottom row of Fig. 5, showing centerline profiles for velocity, temperature, and pressure. In particular, it can be see that the entrainment of colder fluid into the core-region further reduces the temperature and pressure region that is formed at a distance away from the reflected shock (see black lines in Fig. 5). 

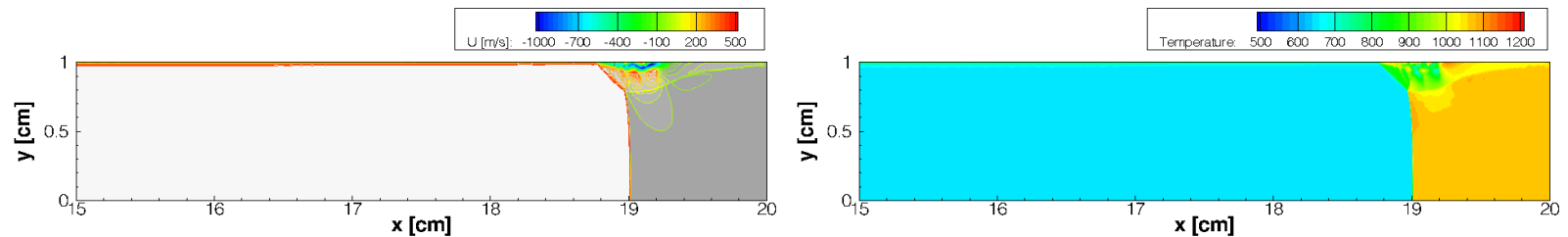

(a) time $t=24.4 \mu \mathrm{s}$.
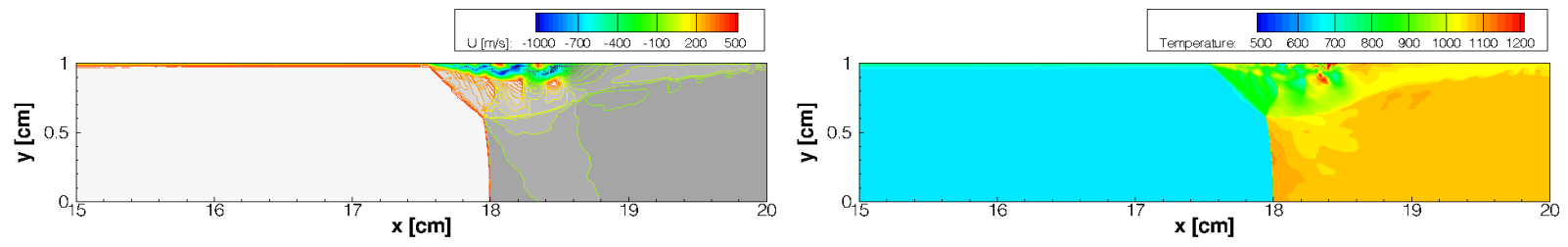

(b) time $t=48.9 \mu \mathrm{s}$.
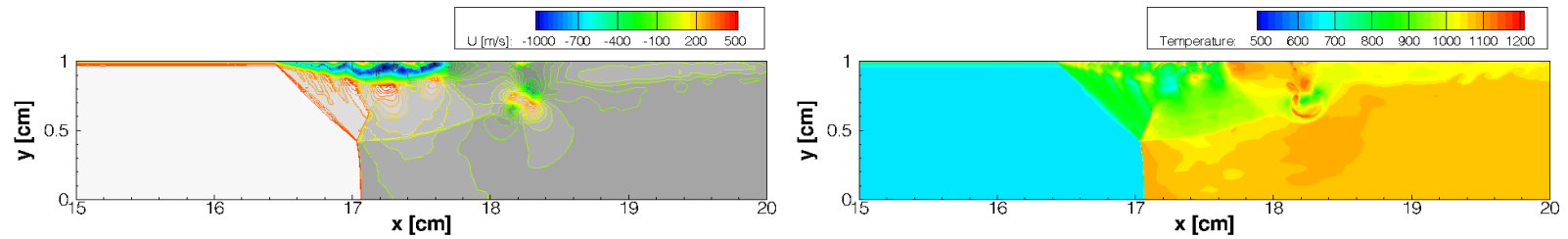

(c) time $t=71.0 \mu \mathrm{s}$
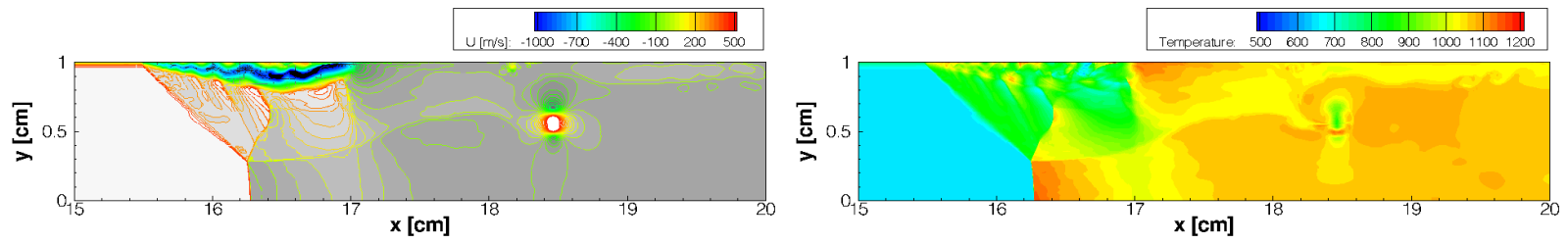

(d) time $t=89.1 \mu \mathrm{s}$.

Figure 4. Instantaneous flow-field structure for velocity (left) and temperature (right) at four time-instance for non-reacting configuration.

\section{B. Reacting Shock Bifurcation}

In the simulation that was presented in the previous section, the chemical source term was set to zero, suppressing all chemical reactions and heat-release. This allowed us to investigate effects of viscous heating and thermal mixing on the temperature of the shock-compressed fluid behind the reflected shock. This section considers the corresponding reacting configuration in order to study the ignition mechanisms and the influence of the bifurcation-induced inhomogeneities on the chemical induction and combustion. Simulation results from this investigation are presented in Figs. 6 and 7, and results at the same time-instances as for the non-reacting case are shown.

Until about $30 \mu$ s after the shock is reflected at the end-wall, the velocity and temperature fields between both non-reacting and reacting simulations are nearly identical (compare red curves in Figs. 5 and 7). However, the entrainment of the boundary-layer fluid and the mixing with the shock-compressed fluid introduces strong velocity fluctuations in the near-wall region, resulting in enhanced viscous heating and temperature increase. The localized hot spots are confined to the region between the tail shock and stagnation point of the ingested and separated boundary layer. This can be seen from the temperature field in Fig. 6(b). At the next time-instance, Fig. 6(c), it can be seen that the boundary layer behind the reflected shock is fully ignited. Measurements of pressure and temperature inside the boundary layer (see blue curves in the top row of Fig. 7) indicate that this ignition process occurs under deflagrative conditions, and the presence of any shock/flame interaction is not evident.

The volumetric ignition in the boundary layer transitions to a detonation wave which originates near the end-wall of the shock tube in the region of prolonged induction time. The detonation wave propagates 

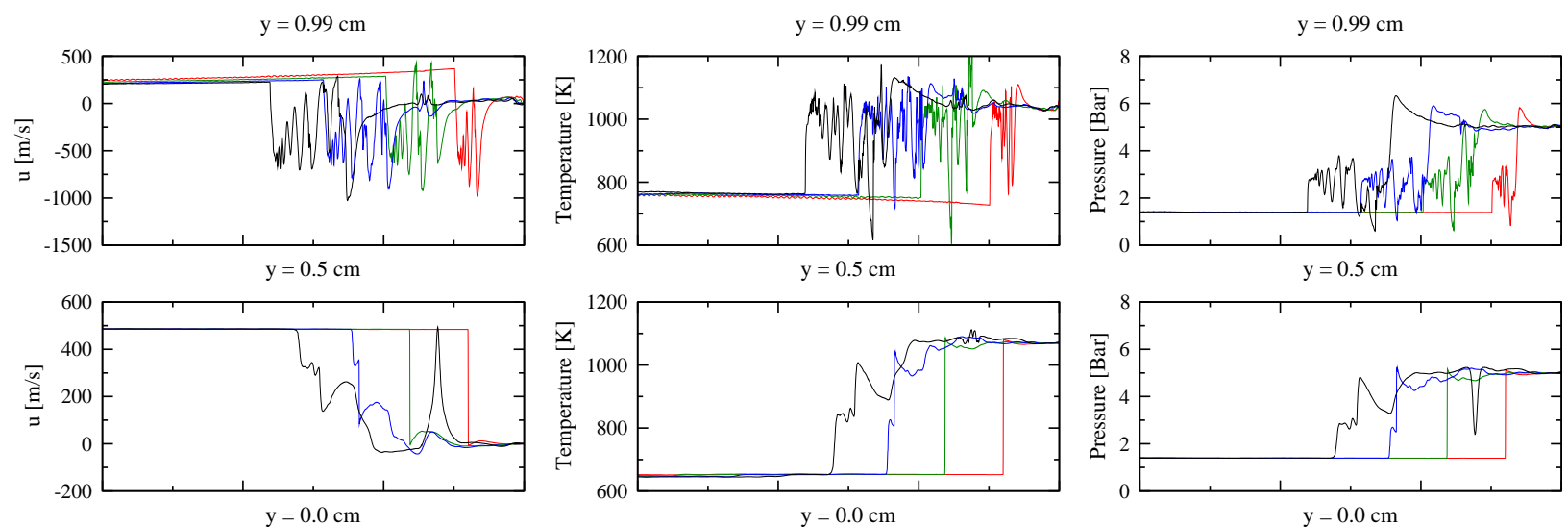

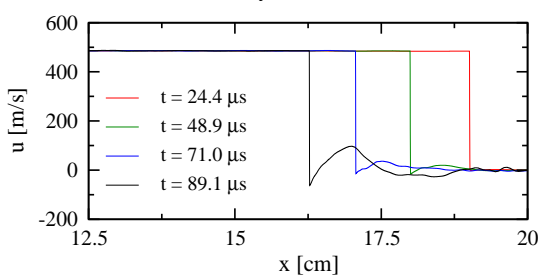

(a) Axial velocity.

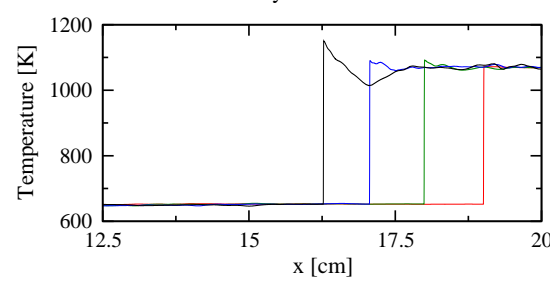

(b) Temperature.

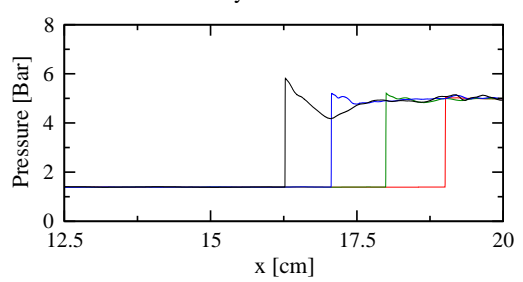

(c) Pressure.

Figure 5. Axial profiles of (a) velocity, (b) temperature, and (c) pressure at different time-instances and three different radial locations along the shock-tube for non-reacting configuration; top: $y=0.99 \mathrm{~cm}$ (boundary layer); middle: $y=0.5 \mathrm{~cm}$; and bottom: $y=0 \mathrm{~cm}$ (centerline).

away from the walls toward the unburned mixture in the core-region, and Fig. 6(d) shows a snapshot of the instantaneous velocity and temperature fields. As the detonation waves propagates toward the reflected shock, a Mach-reflection pattern develops.

After the detonation, temperature and pressure of the double-shock compressed reacted fluid increase, and the detonation structure is visible in the cross-sectional plots in Fig. 7 (see black curves). Interesting is also the formation of a secondary ignition kernel in the region between the reflected shock and the outwardpropagating detonation front. This kernel eventually expands and competes with the primary detonation wave for the consumption of unburned reactants.

\section{Conclusions}

Detailed simulations of the interaction of a reflected shock with the boundary layer that is formed behind the incident shock-wave are performed. In these investigations, the end-section of a shock-tube is considered, and the simulations are performed using an adaptive mesh-refinement (AMR) technique to resolve all relevant physical scales that are associated with the viscous boundary-layer, the reaction front, and the shock-wave. The AMR-method uses a hierarchy of structured and embedded grids that are successively refined. Five AMR-levels are employed, resulting in a minimum grid size below $10 \mu \mathrm{m}$. A hybrid shockcapturing WENO/central differencing scheme with sixth-order accurate spatial discretization is used, and the reactive Navier-Stokes equations are solved under consideration of detailed reaction chemistry and multicomponent diffusive transport.

In the present study the shock-compression and ignition of an Argon-diluted hydrogen/oxygen mixture, experimentally investigated by Blumenthal et al. ${ }^{18,51}$ and Pang et al. ${ }^{54}$ at target conditions of $p_{5}=5$ bar and $T_{5}=1100 \mathrm{~K}$ was considered; the reaction chemistry is described using the updated high-pressure $\mathrm{H}_{2} / \mathrm{O}_{2}$ kinetic mechanism of Burke et al. ${ }^{55}$ The simulation of the shock-compression of the non-reacting mixture shows the instantaneous formation of a bifurcation region, ingesting fluid from the upstream boundary-layer into the stagnation region. The mixing of the thermally stratified fluid, carrying different momentum and enthalpy, introduces inhomogeneities in the core-region behind the reflected shock. In simulations of the reacting mixture it was shown that these inhomogeneities act as ignition kernels that are located in the near- 

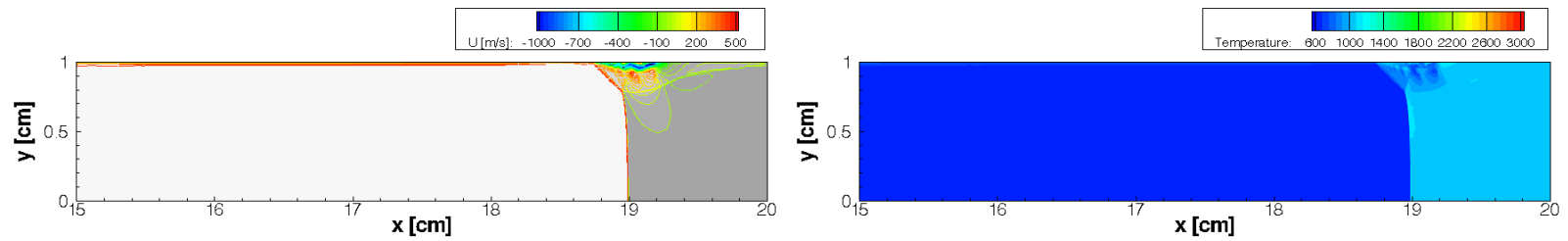

(a) time $t=24.4 \mu \mathrm{s}$.
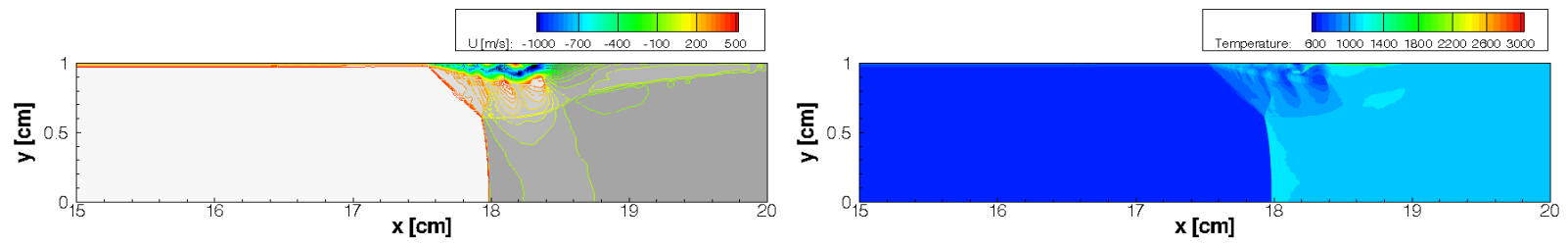

(b) time $t=48.9 \mu \mathrm{s}$.
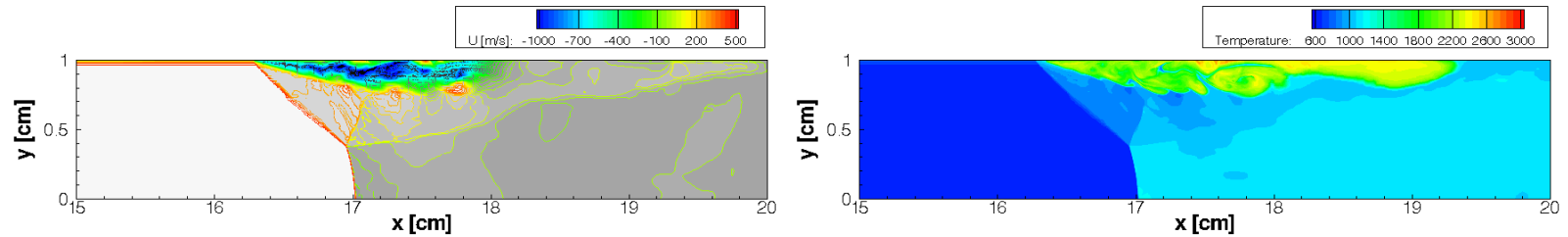

(c) time $t=71.0 \mu \mathrm{s}$.
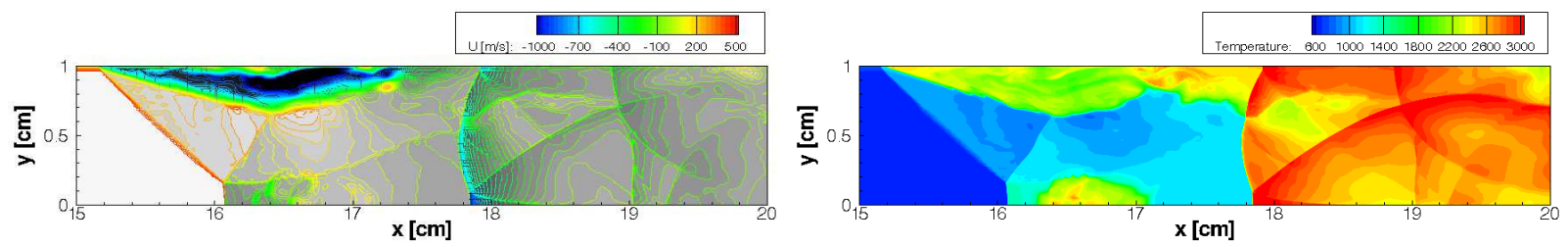

(d) time $t=89.1 \mu \mathrm{s}$.

Figure 6. Instantaneous flow-field structure for velocity (left) and temperature (right) at four time-instance for reacting configuration. Time $t$ is measured after the shock is reflected at the end wall.

wall region. During the induction period, these kernels slowly expand and eventually transition to a strong detonation wave that rapidly consumes the unburned mixture. In competition with this detonation wave are the presence of secondary ignition kernels that appear in the unreacted core-region between reflected shock and detonation wave. These detailed simulations confirm at two-stage ignition process that is characteristic for the weak-ignition.

\section{Acknowledgments}

The authors gratefully acknowledge financial support through the Air Force Office of Scientific Research under Award No. FA9550-11-1-0031 with Chiping Li as program manager.

\section{References}

${ }^{1}$ Duff, R. E., "Shock-tube performance at low initial pressure," Phys. Fluids, Vol. 2, No. 2, 1959, pp. $207-216$.

${ }^{2}$ White, D. R., "Influence of diaphragm opening time on shock-tube flows," J. Fluid Mech., Vol. 4, 1958, pp. 585-599.

${ }^{3}$ Miller, C. G. and Jones, J. J., "Incident shock wave characteristics in air, argon, carbon dioxide, and helium in a shock tube with unheated helium driver," Tech. Rep. TN D-8099, NASA Langley Research Center Hampton, 1975.

${ }^{4}$ Nagamatsu, H. T., Geiger, R. E., and Sheer, R. E., "Hypersonic shock tunnel," Am. Rocket Society J., Vol. 29, No. 5, 1959, pp. 332-340.

${ }^{5}$ Outa, E., Tajima, K., and Hayakawa, K., "Shock tube flow influenced by diaphragm opening (two-dimensional flow near the diaphragm)," Proceedings of the International Shock Tube Symposium, Vol. 10, 1975, pp. 312-319. 

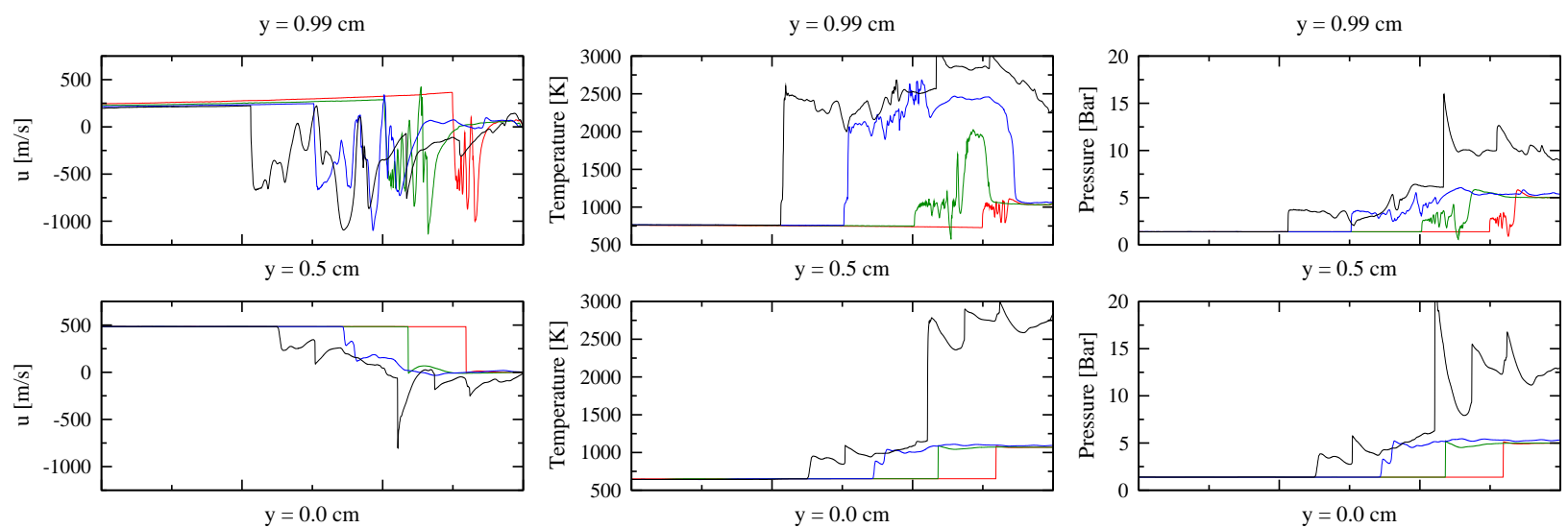

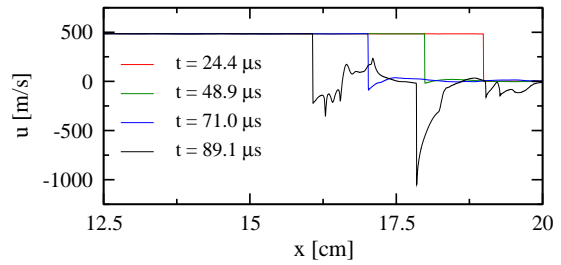

(a) Axial velocity.

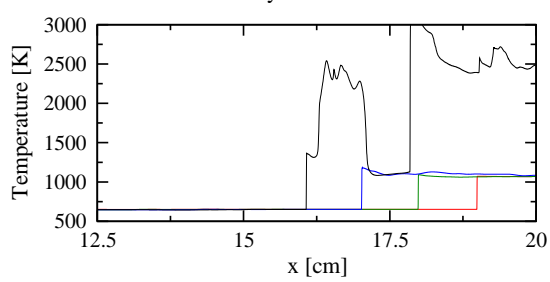

(b) Temperature.

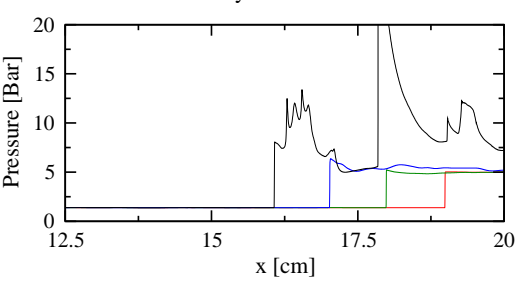

(c) Pressure.

Figure 7. Axial profiles of (a) velocity, (b) temperature, and (c) pressure at different time-instances and three different radial locations along the shock-tube for reacting configuration; top: $y=0.99 \mathrm{~cm}$ (boundary layer); middle: $y=0.5 \mathrm{~cm}$; and bottom: $y=0 \mathrm{~cm}$ (centerline).

${ }^{6}$ Belford, R. L. and Strehlow, R. A., "Shock tube techniques in chemical kinetics," Ann. Rev. Phys. Chem., Vol. 20, 1969, pp. $247-272$

${ }^{7}$ Petersen, E. L. and Hanson, R. K., "Nonideal effects behind reflected shock waves in a high-pressure shock tube," Shock Waves, Vol. 10, 2001, pp. 405-420.

${ }^{8}$ Petersen, E. L., A Shock Tube and Diagnostics for Chemistry Measurements at Elevated Pressures with Application to Methane Ignition, Ph.D. thesis, Stanford University, 1998.

${ }^{9}$ Mark, H., "The interaction of a reflected shock wave with the boundary layer in a shock tube," J. Aeronaut. Sci., Vol. 24, 1957, pp. 304-306.

${ }^{10}$ Strehlow, R. A. and Cohen, A., "Limitations of the reflected shock technique for studying fast chemical reactions and its application to the observation of relaxation in nitrogen and oxygen," J. Chem. Phys., Vol. 30, 1959, pp. $257-265$.

${ }^{11}$ Center, R., "Reflected shock interaction with shock tube boundary layers," Phys. Fluids, Vol. 6, 1963, pp. 307-308.

${ }^{12}$ Dyner, H. B., "Density variation due to reflected shock-boundary layer interaction," Phys. Fluids, Vol. 9, 1966, pp. 879892.

${ }^{13}$ Honda, M., Takayama, K., Onodera, O., and Kohama, Y., "Motion of reflected shock waves in shock tube," Proceedings of the International Shock Tube Symposium, Vol. 10, 1975, pp. 312-319.

${ }^{14}$ Fokeev, V. P. and Gvozdeva, L. G., "Study of bifurcation of reflected shock waves in channels of various crosssections," Current topics in shock waves 17th international symposium on shock waves and shock tube. AIP Conference Proceedings, Vol. 208, 1990, pp. 862-866.

${ }^{15}$ Kleine, H., Lyakhov, V. N., Gvozdeva, L. G., and Grönig, H., "Bifurcation of a reflected shock wave in a shock tube," Shock waves; Proceedings of the 18th International Symposium, Vol. 1, 1991, pp. 261-266.

${ }^{16}$ Chaos, M. and Dryer, F. L., "Chemical-kinetic modeling of ignition delay: Considerations in interpreting shock tube data," Int. J. Chem. Kinet., Vol. 42, 2010, pp. 143-150.

${ }^{17}$ Pfahl, U., Fieweger, K., and Adomeit, G., "Self-ignition of diesel-relevant hydrocarbon-air mixtures under engine conditions," Proc. Combust. Inst., Vol. 26, 1996, pp. 781-789.

${ }^{18}$ Blumenthal, R., Fieweger, K., Komp, K. H., and Adomeit, G., "Gas dynamic features of self ignition of non diluted fuel/air mixtures at high pressure," Combust. Sci. Tech., Vol. 113, 1996, pp. 137-166.

${ }^{19}$ Cadman, P., Bambrey, R. J., Box, S. K., and Thomas, G. O., "Ethylene combustion studied over a wide temperature range in high-temperature shock waves," Combust. Sci. Tech., Vol. 174, 2002, pp. 111-127.

${ }^{20}$ Meyer, J. W. and Oppenheim, A. K., "On the shock-induced ignition of explosive gases," Proc. Combust. Inst., Vol. 13, 1971, pp. 1153-1164.

${ }^{21}$ Oppenheim, A. K., "Dynamic features of combustion," Phil. Trans. Roy. Soc. London, Vol. 315, 1985, pp. 471-508.

${ }^{22}$ Vermeer, D. J., Meyer, J. W., and Oppenheim, A. K., "Auto-ignition of hydrocarbons behind reflected shock waves," Combust. Flame, Vol. 18, 1972, pp. 327-336. 
${ }^{23}$ Oran, E. S. and Boris, J. P., "Weak and strong ignition. II. Sensitivity of the hydrogen-oxygen system," Combust. Flame, Vol. 48, 1982, pp. 149-161.

${ }^{24}$ Oran, E. S., Young, J. P., Boris, J. P., and Cohen, A., "Weak and strong ignition. I. Numerical simulations of shock tube experiments," Combust. Flame, Vol. 48, 1982, pp. 135-148.

${ }^{25}$ T., B. C. and K., H. R., "Shock tube measurements of rate coefficients of elementary gas reactions," J. Phys. Chem., Vol. 83, No. 6, 1979, pp. 757-763.

${ }^{26}$ Davidson, D. F. and Hanson, R. K., "Recent advances in shock tube/laser diagnostic methods for improved chemical kinetics measurements," Shock Waves, Vol. 19, 2009, pp. 271-283.

${ }^{27}$ Michael, J. V. and Sutherland, J. W., "The thermodynamic state of the hot gas behind reflected shock-waves - Implication to chemical-kinetics," Int. J. Chem. Kinet., Vol. 18, 1986, pp. 409-436.

${ }^{28}$ Lifshitz, A., Bar-Nun, A., de Boer, P. C. T., and Resler, Jr., E. L., "Boundary Layer Effects on Chemical Kinetics Studies in a Shock Tube," J. Chem. Phys., Vol. 53, 1970, pp. 3050-3055.

${ }^{29}$ Khandelwal, S. C. and Skinner, G. B., "Shock tube studies of hydrocarbon oxidation," Shock Waves in Chemistry, edited by A. Lifshitz, Marcel Dekker, Inc., 1981, pp. 1-57.

${ }^{30}$ Lifshitz, A., Shock Waves in Chemistry, Marcel Dekker, Inc., 1981.

${ }^{31}$ Daru, V. and Tenaud, C., "Numerical simulation of the viscous shock tube problem by using a high resolution monotonicity-preserving scheme," Computers \& Fluids, Vol. 38, 2009, pp. 664-676.

${ }^{32}$ Frenklach, M., Li Kwok Cheong, C. K., and Oran, E. S., "LDV measurements of gas flow behind reflected shocks," Dynamcis of Flame and Reactive Systems, edited by J. R. Bowen, N. Manson, A. K. Oppenheim, and R. I. Soloukhin, Vol. 95 of Progress in Astronautics and Aeronautics, 1984, pp. 722-735.

${ }^{33}$ Perkins, A. N., Ramsey, M., Pitz, R. W., Wehrmeyer, J. A., and Nelius, A. E., "Investigation of a bow shock in a shock tube flow facility using hydroxyl tagging velocimetry (HTV)," AIAA Paper 2011-1092, 2011.

${ }^{34}$ Sanderson, R. J., "Interpretation of pressure measurements behind the reflected shock in a rectangular shock tube," AIAA J., Vol. 7, 1969, pp. 1370-1372.

${ }^{35}$ Yoo, J., Mitchell, D., Davidson, D. F., and Hanson, R. K., "Planar laser-induced fluorescence imaging in shock tube flows," Exp. Fluids, Vol. 49, No. 4, 2010, pp. 751-759.

${ }^{36}$ Yoo, J., Mitchell, D., Davidson, D. F., and Hanson, R. K., "Near-wall imaging using toluene-based planar laser-induced fluorescence in shock tube flow," Shock Waves, Vol. 21, 2011, pp. 523-532.

${ }^{37}$ Berger, M. J. and Colella, P., "Local adaptive mesh refinement for shock hydrodynamics," J. Comp. Phys., Vol. 82, 1989, pp. $64-84$.

${ }^{38}$ Deiterding, R., "AMROC - Blockstructured Adaptive Mesh Refinement in Object-oriented C++," Available at http://amroc.sourceforge.net, 2008.

${ }^{39}$ Bell, J., Berger, M., Saltzman, J., and Welcome, M., "Three-dimensional adaptive mesh refinement for hyperbolic conservation laws," SIAM J. Sci. Comput., Vol. 15, No. 1, 1994, pp. 127-138.

${ }^{40}$ Deiterding, R., Parallel adaptive simulation of multi-dimensional detonation structures, Ph.D. thesis, Brandenburgische Technische Universität Cottbus, 2003.

${ }^{41}$ Deiterding, R., "Construction and application of an AMR algorithm for distributed memory computers," Adaptive Mesh Refinement - Theory and Applications, edited by T. Plewa, T. Linde, and V. G. Weirs, Vol. 41 of Lecture Notes in Computational Science and Engineering, Springer, 2005, pp. 361-372.

${ }^{42}$ Parashar, M. and Browne, J. C., "On partitioning dynamic adaptive grid hierarchies," Proc. of the 29th Annual Hawaii Int. Conf. on System Sciences, 1996, pp. 604-613.

${ }^{43}$ Parashar, M. and Browne, J. C., "System engineering for high performance computing software: The HDDA/DAGH infrastructure for implementation of parallel structured adaptive mesh refinement," Structured Adaptive Mesh Refinement Grid Methods, IMA Volumes in Mathematics and its Applications, Springer, 1997, pp. 1-18.

${ }^{44}$ Jiang, G. S. and Shu, C. W., "Efficient implementation of weighted ENO schemes," J. Comp. Phys., Vol. 126, 1996, pp. 202-228.

${ }^{45} \mathrm{Shu}, \mathrm{C} . \mathrm{W} .$, "Essentially non-oscillatory and weighted essentially non-oscillatory schemes for hyperbolic conservation laws," Tech. Rep. No. 97-65, Institute for Computer Applications in Science and Engineering NASA Langley Research Center, 1997.

${ }^{46}$ Hill, D. J. and Pullin, D. I., "Hybrid tuned center-difference-WENO method for large eddy simulations in the presence of strong shocks," J. Comp. Phys., Vol. 194, 2004, pp. 435-450.

${ }^{47}$ Pantano, C., Deiterding, R., Hill, D. J., and Pullin, D. I., "A low numerical dissipation patch-based adaptive mesh refinement method for large-eddy simulation of compressible flows," J. Comp. Phys., Vol. 221, 2007, pp. 63-87.

${ }^{48}$ Honein, A. E. and Moin, P., "Higher entropy conservation and numerical stability of compressible turbulence simulations," J. Comp. Phys., Vol. 201, 2004, pp. 531-545. 2008.

${ }^{49}$ Lombardini, M., Richtmyer-Meshkov instability in converging geometries, Ph.D. thesis, California Institute of Technology,

${ }^{50}$ Yetter, R. A., Rabitz, H., and Hedges, R. M., "A combined stability-sensitivity analysis of weak and strong reactions of hydrogen/oxygen mixtures," Int. J. Chem. Kinet., Vol. 23, 1991, pp. 251-278.

${ }^{51}$ Blumenthal, R., Fieweger, K., Komp, K. H., Adomeit, G., and Gelfand, B. E., "Self-ignition of $\mathrm{H}_{2}$-air mixtures at high pressure and low temperature," Proc. Int. Symp. Shock Waves, Vol. 20, 1995, pp. 935-940.

${ }^{52}$ Wang, B. L., Olivier, H., and Gröning, H., "Ignition of shock-heated $\mathrm{H}_{2}$-air-steam mixtures," Combust. Flame, Vol. 133, 2003, pp. 93-106.

${ }^{53}$ Chaos, M. and Dryer, F. L., "Syngas combustion kinetics and applications," Combust. Sci. Tech., Vol. 180, 2008, pp. 1053-1096.

${ }^{54}$ Pang, G. A., Davidson, D. F., and Hanson, R. K., "Experimental study and modeling of shock tube ignition delay times for hydrogen/oxygen/argon mixtures at low temperatures," Proc. Combust. Inst., Vol. 32, 2009, pp. 181-188. 
${ }^{55}$ Burke, M. P., Chaos, M., Ju, Y., Dryer, F. L., and Klippenstein, S. J., "Comprehensive $\mathrm{H}_{2} / \mathrm{O}_{2}$ kinetic model for high-pressure combustion," Int. J. Chem. Kinet., Vol. 44, No. 7, 2012, pp. 444-474. 